\title{
CMA and Soft Decision-Directed Scheme for Semi-Blind Beamforming of QAM Systems
}

\author{
S. Chen, W. Yao and L. Hanzo \\ School of Electronics and Computer Science \\ University of Southampton, Southampton SO17 1BJ, UK \\ E-mails: $\{$ sqc,wy07r,lh\} @ecs.soton.ac.uk
}

\begin{abstract}
We propose a semi-blind adaptive beamforming approach for wireless systems that employ high-throughput quadrature amplitude modulation signalling schemes. A minimum number of training symbols, equal to the number of receive antenna-array's elements, are first utilised to provide a rough initial least squares estimate of the beamformer's weight vector. A concurrent constant modulus algorithm and soft decisiondirected scheme, originally developed for single-user blind channel equalisation, is then applied to adapt the beamformer. It is demonstrated that this semi-blind adaptive beamforming scheme is capable of converging fast to the minimum mean-square-error beamforming solution.
\end{abstract}

\section{INTRODUCTION}

The ever-increasing demand for wireless communication capacity has motivated the development of antenna array assisted spatial processing techniques [1], [2], [3], [4], [5], [6], [7] in order to further improve the achievable spectral efficiency. In particular, adaptive beamforming with antenna arrays [2], [5], [7] has shown real promise in achieving substantial capacity enhancement. This is because adaptive beamforming is capable of separating signals transmitted on the same carrier frequency, and thus provides a practical means of supporting multiusers in a space division multiple access scenario. In order to further improve the achievable bandwidth efficiency, high-throughput quadrature amplitude modulation (QAM) schemes [8] has become popular in numerous wireless network standards. For example, the 16-QAM and 64-QAM schemes were adopted in the WiMax standard [9]. The classical beamforming design is the minimum mean square error (MMSE) solution, which can be realised using the trainingbased adaptive algorithms [5], [10], [11], [12]. Pure trainingbased schemes however require a high propotion of training symbols, which considerably reduces the achievable system throughput. Pure blind beamforming [13], [14], [15], [16] does not reduce the achievable system throughput at the expense of high computational complexity and slow convergence. Moreover, blind beamforming results in unavoidable estimation and decision ambiguities [17], [18].

An effective means of resolving the estimation and decision ambiguities inherented in blind schemes is to employ a few training symbols. Combining a very short training with blind adaptive beamforming leads to attractive semi-blind adaptive beamforming [19], [20], [21], [22]. This contribution proposes a low-complexity semi-blind adaptive beamforming algorithm.
In particular, we consider adaptive beamforming assisted receiver for wireless systems that employ high-throughput QAM signalling [8]. As we will consider the low-complexity MMSE solution as the optimal design for beamforming, the number of users supported by the system is assumed to be no more than the number of receive antenna array's elements. The proposed adaptive beamforming method is semi-blind as we employ a minimum number of pilots, which is equal to the number of receiver array elements, to provide a rough initial least squares (LS) estimate for the beamformer's weight vector. In general, this initialisation is not sufficiently accurate to achieve an "opening-eye" and, therefore, it is unsafe to carry out decisiondirected (DD) adaptation for the beamformer. However, we can apply a constant modulus algorithm (CMA) assisted soft DD (SDD) blind adaptive algorithm to adapt the beamformer. The concurrent CMA and SDD algorithm was originally derived for blind equalisation of single-input single-output QAM systems [23], and it was extended to single-input multipleoutput systems in [24]. This blind adaptive scheme has a very low computational complexity. In the present semi-blind beamforming application, owing to the initial information provided by the training pilots, the algorithm converges much faster than the pure blind adaptation case, and it is capable of approaching the performance of the MMSE beamforming solution based on the perfect channel knowledge, as will be shown in our simulation study.

We adopt the following notational conventions throughout this contribution. Boldface capitals and lower-case letters stand for matrices and vectors, respectively, while $\mathbf{I}_{K}$ denotes the $K \times K$ identity matrix. Furthermore, $(\bullet)^{T}$ and $(\bullet)^{H}$ are the transpose and Hermitian operators, respectively, while $\|\bullet\|$ and $|\bullet|$ denote the norm and magnitude operators, respectively. $E[\bullet]$ is the expectation operator, while $(\bullet)^{*}$ denotes the complex conjugate. Finally, $j=\sqrt{-1}$.

\section{BEAMFORMING MODEL}

We consider a coherent communication system that supports $n_{T}$ users, where each user transmits a $M$-QAM signal on the same angular carrier frequency of $\omega$. In order to achieve user separation in the angular domain [6], [25], the receiver is equipped with a linear antenna array consisting of $n_{R}$ uniformly spaced elements. Further assume that the communication is over flat fading channels. Then the system 
is described by the following model

$$
\mathbf{x}(k)=\mathbf{H} \mathbf{s}(k)+\mathbf{n}(k),
$$

where $k$ denotes the symbol index, $\mathbf{x}(k)=$ $\left[x_{1}(k) \quad x_{2}(k) \cdots x_{n_{R}}(k)\right]^{T}$ is the received signal vector, $\mathbf{n}(k)=\left[n_{1}(k) n_{2}(k) \cdots n_{n_{R}}(k)\right]^{T}$ is the complex-valued Gaussian white noise vector associated with the system with $E\left[\mathbf{n}(k) \mathbf{n}^{H}(k)\right]=2 \sigma_{n}^{2} \mathbf{I}_{n_{R}}, \mathbf{s}(k)=\left[s_{1}(k) s_{2}(k) \cdots s_{n_{T}}(k)\right]^{T}$ is the transmitted symbols vector of the $n_{T}$ users with the symbol energy given by $E\left[\left|s_{m}(k)\right|^{2}\right]=\sigma_{s}^{2}$ for $1 \leq m \leq n_{T}$, and $\mathbf{H}$ denotes the $n_{R} \times n_{T}$ complex-valued system channel matrix.

More specifically, the system channel matrix $\mathbf{H}=\left[h_{l, m}\right]$, where $1 \leq l \leq n_{R}$ and $1 \leq m \leq n_{T}$, is defined by

$$
\mathbf{H}=\left[\mathbf{h}_{1} \mathbf{h}_{2} \cdots \mathbf{h}_{n_{T}}\right]=\left[\begin{array}{lll}
A_{1} \boldsymbol{\eta}_{1} & A_{2} \boldsymbol{\eta}_{2} \cdots A_{n_{T}} \boldsymbol{\eta}_{n_{T}}
\end{array}\right],
$$

where $A_{m}$ denotes the non-dispersive complex-valued channel coefficient for user $m$, and the steering vector for user $m$ is given by

$$
\boldsymbol{\eta}_{m}=\left[e^{j \omega t_{1}\left(\theta_{m}\right)} e^{j \omega t_{2}\left(\theta_{m}\right)} \cdots e^{j \omega t_{n_{R}}\left(\theta_{m}\right)}\right]^{T}
$$

with $\theta_{m}$ being the angle of arrival for user $m$ and $t_{l}\left(\theta_{m}\right)$ being the relative time delay at array element $l$ for user $m$. $\theta_{m}$ is uniformly distributed in $[0,2 \pi)$ and the magnitude of $A_{m}$ is a Rayleigh process. However, the fading is assumed to be sufficiently slow, so that during the time period of a transmission block or frame, all the related entries $h_{l, m}$ in the system channel matrix $\mathbf{H}$ is deemed unchanged. From frame to frame, $h_{l, m}$ are assumed to be uncorrelated complex-valued Gaussian processes with zero mean and $E\left[\left|h_{l, m}\right|^{2}\right]=1$.

The modulation scheme is the $M$-QAM and, therefore, the transmitted data symbols $s_{m}(k), 1 \leq m \leq n_{T}$, take the values from the $M$-QAM symbol set defined by

$$
\mathcal{S} \triangleq\left\{s_{i, q}=u_{i}+j u_{q}, 1 \leq i, q \leq \sqrt{M}\right\}
$$

with the real-part symbol $\Re\left[s_{i, q}\right]=u_{i}=2 i-\sqrt{M}-1$ and the imaginary-part symbol $\Im\left[s_{i, q}\right]=u_{q}=2 q-\sqrt{M}-1$. The average signal-to-noise ratio (SNR) of the system is defined as $\mathrm{SNR}=n_{T} \times \sigma_{s}^{2} / 2 \sigma_{n}^{2}$. Without the loss of generality, user one is assumed to be the desired user and the rest of the users are interfering ones. A beamformer

$$
y(k)=\mathbf{w}^{H} \mathbf{x}(k)
$$

is used to detect the transmitted symbols $s_{1}(k)$ of the desired user, where $\mathbf{w}=\left[\begin{array}{llll}w_{1} & w_{2} & \cdots & w_{n_{R}}\end{array}\right]^{T}$ is the complex-valued weight vector of the beamformer. With the perfect channel knowledge, the optimal MMSE solution that minimises the mean square error criterion $E\left[\left|s_{1}(k)-y(k)\right|^{2}\right]$ is given by

$$
\mathbf{w}_{\text {MMSE }}=\left(\mathbf{H H}^{H}+\frac{2 \sigma_{n}^{2}}{\sigma_{s}^{2}} \mathbf{I}_{n_{R}}\right)^{-1} \mathbf{h}_{1} .
$$

\section{The Proposed Semi-Blind Algorithm}

Let the number of available training symbols be $K$, and denote the available training data as $\mathbf{X}_{K}=[\mathbf{x}(1) \mathbf{x}(2) \cdots \mathbf{x}(K)]$ and $\mathbf{s}_{K}=\left[s_{1}(1) s_{1}(2) \cdots s_{1}(K)\right]^{T}$. The LS estimate of the beamformer's weight vector based on the training data $\left\{\mathbf{X}_{K}, \mathbf{s}_{K}\right\}$ is readily given as

$$
\mathbf{w}(0)=\left(\mathbf{X}_{K} \mathbf{X}_{K}^{H}\right)^{-1} \mathbf{X}_{K} \mathbf{s}_{K}^{*} .
$$

In order to maintain throughput, the number of training pilots should be as small as possible. To ensure that $\mathbf{X}_{K} \mathbf{X}_{K}^{H}$ has a full rank, we will choose $K=n_{R}$ as the minimum number of training symbols. Because the training data with $K=n_{R}$ are insufficient, the initial LS weight vector (7) may not be sufficiently accurate to open the eye. Therefore, DD adaptation is generally unsafe. However, we can apply the concurrent CMA and SDD blind scheme [23], [24] to adapt the beamformer (5) with $\mathbf{w}(0)$ of (7) as the initial weight vector. Let the beamformer's weight vector be split into two parts, yielding $\mathbf{w}=\mathbf{w}_{c}+\mathbf{w}_{d}$, and denote the beamformer's output at sample $k$ as $y(k)=\mathbf{w}^{H}(k) \mathbf{x}(k)$. The initial $\mathbf{w}_{c}$ and $\mathbf{w}_{d}$ are simply set to $\mathbf{w}_{c}(0)=\mathbf{w}_{d}(0)=0.5 \mathbf{w}(0)$.

Specifically the weight vector $\mathbf{w}_{c}$ is updated using the classical CMA [26], [27]

$$
\left.\begin{array}{l}
\varepsilon(k)=y(k)\left(\Delta-|y(k)|^{2}\right), \\
\mathbf{w}_{c}(k+1)=\mathbf{w}_{c}(k)+\mu_{\mathrm{CMA}} \varepsilon^{*}(k) \mathbf{x}(k),
\end{array}\right\}
$$

where $\Delta=E\left[\left|s_{1}(k)\right|^{4}\right] / E\left[\left|s_{1}(k)\right|^{2}\right]$ and $\mu_{\mathrm{CMA}}$ is the step size of the CMA. The weight vector $\mathbf{w}_{d}$ by contrast is updated using the SDD scheme [23], [24], which has its root in the blind equalisation scheme of [28]. The complex phasor plane is divided into the $M / 4$ rectangular regions, and each region $\mathcal{S}_{i, l}$ contains four symbol points as defined in the following

$$
\mathcal{S}_{i, l}=\left\{s_{p, q}, p=2 i-1,2 i, q=2 l-1,2 l\right\},
$$

where $1 \leq i, l \leq \sqrt{M} / 2$. An illustration of this local decision region is illustrated in Fig. 1. If the beamformer's output $y(k) \in \mathcal{S}_{i, l}$, a local approximation of the marginal probability density function (PDF) of $y(k)$ is given by [23], [24]

$$
\hat{p}(\mathbf{w}, y(k)) \approx \sum_{p=2 i-1}^{2 i} \sum_{q=2 l-1}^{2 l} \frac{1}{8 \pi \rho} e^{-\frac{\left|y(k)-s_{p, q}\right|^{2}}{2 \rho}},
$$

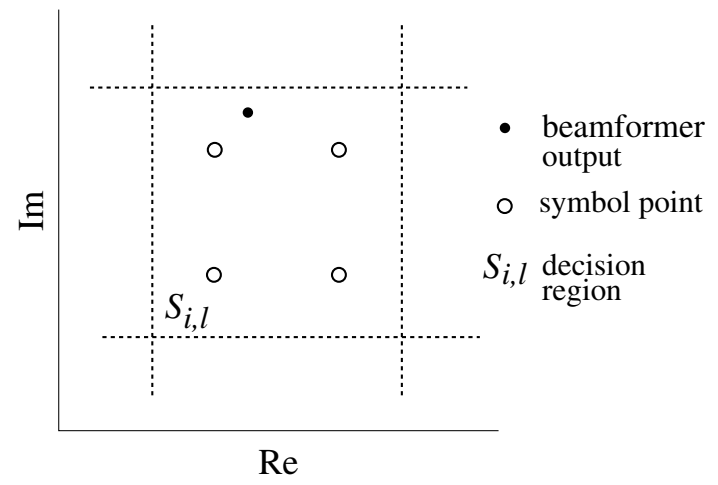

Fig. 1. Illustration of local decision regions for the soft decision-directed adaptation procedure for QAM constellation. 
where $\rho$ defines the cluster width associated with the four clusters of each region $\mathcal{S}_{i, l}$. The SDD algorithm is designed to maximise the $\log$ of the local marginal PDF criterion $E\left[J_{\mathrm{LMAP}}(\mathbf{w}, y(k))\right]$, where $J_{\mathrm{LMAP}}(\mathbf{w}, y(k))=$ $\rho \log (\hat{p}(\mathbf{w}, y(k)))$, via a stochastic gradient optimisation. Specifically, $\mathbf{w}_{d}$ is updated according to [23], [24]

$$
\mathbf{w}_{d}(k+1)=\mathbf{w}_{d}(k)+\mu_{\mathrm{SDD}} \frac{\partial J_{\mathrm{LMAP}}(\mathbf{w}(k), y(k))}{\partial \mathbf{w}_{d}},
$$

where $\mu_{\mathrm{SDD}}$ is the step size of the SDD, and

$$
\begin{gathered}
\frac{\partial J_{\operatorname{LMAP}}(\mathbf{w}, y(k))}{\partial \mathbf{w}_{d}}= \\
\frac{1}{Z_{N}} \sum_{p=2 i-1}^{2 i} \sum_{q=2 l-1}^{2 l} e^{-\frac{\left|y(k)-s_{p, q}\right|^{2}}{2 \rho}}\left(s_{p, q}-y(k)\right)^{*} \mathbf{x}(k),
\end{gathered}
$$

with the normalisation factor

$$
Z_{N}=\sum_{p=2 i-1}^{2 i} \sum_{q=2 l-1}^{2 l} e^{-\frac{\left|y(k)-s_{p, q}\right|^{2}}{2 \rho}} .
$$

The choice of the cluster width $\rho$, defined in the context of the local PDF (10), should ensure a proper separation of the four clusters of $\mathcal{S}_{i, l}$. As the minimum distance between the two neighbouring constellation points is $2, \rho$ is typically chosen to be less than 1 . If the value of $\rho$ is too large, a desired degree of separation may not be achieved. On the other hand, if too small a $\rho$ value is used, the algorithm attempts to impose an overly tight control on the size of clusters and hence may fail to achieve its goal. Apart from these two extreme situations, the performance of the algorithm is not overly sensitive to the value of $\rho$ employed and an appropriate $\rho$ can easily be chosen from a large range of values. More specifically, when the objective of removing interference is accomplished, $y(k) \approx s_{1}(k)+e(k)$, where $e(k)$ is Gaussian distributed with zero mean. Therefore, the value of $\rho$ is related to the variance of $e(k)$, which is $2 \sigma_{n}^{2} \mathbf{w}^{H} \mathbf{w}$. Thus, for high SNR situations, small $\rho$ is desired, while for low SNR cases, large $\rho$ is preferred. Because of the information provided by the training pilots in the form of the initial weight vector (7), smaller $\rho$ can be used, compared with the case of pure blind adaptation in [23], [24], which leads to better steadystate performance. Soft decision nature becomes explicit in (12), because rather than committing to a single hard decision $\mathcal{Q}[y(k)]$, where $\mathcal{Q}[\bullet]$ denotes the quantisation operator, as the hard DD scheme would, alternative decisions are also considered in the local region $\mathcal{S}_{i, l}$ that includes $\mathcal{Q}[y(k)]$, and each tentative decision is weighted by an exponential term $e^{\{\bullet\}}$, which is a function of the distance between the equaliser's soft output $y(k)$ and the tentative decision $s_{p, q}$. This soft decision nature substantially reduces the risk of error propagation and achieves faster convergence, compared with the hard DD scheme [23], [24].

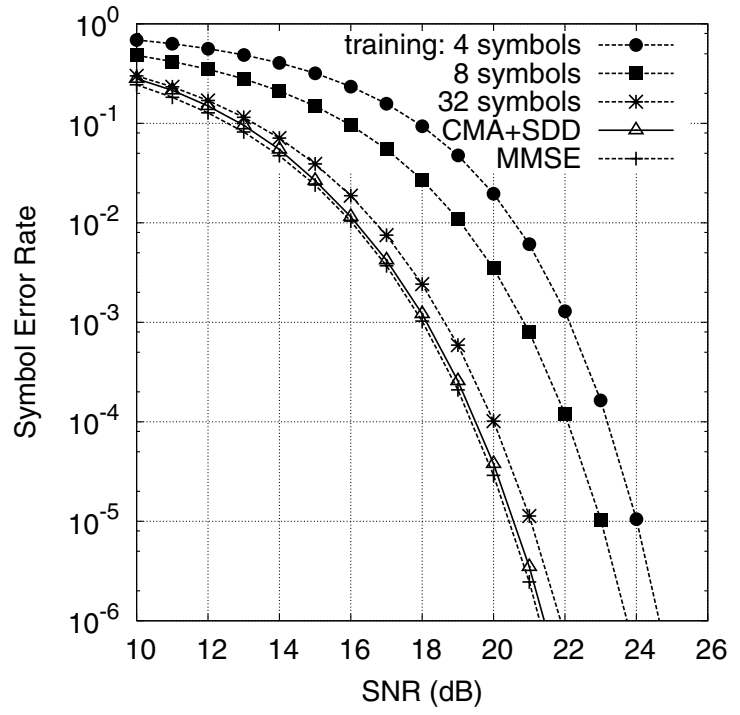

Fig. 2. Desired user-one symbol error rate performance comparison of the proposed semi-blind adaptive beamforming given $K=4$ training symbols, the training-based adaptive beamforming given different numbers of training symbols, and the true MMSE beamforming given perfect channel knowledge, for the stationary system of four-element array supporting four 16-QAM users.

\section{Simulation Study}

A simulation study was carried out to investigate the proposed semi-blind adaptive beamforming scheme based on the concurrent CMA and SDD algorithm. The achievable performance was assessed in the simulation using the symbol error rate (SER). The analytical SER $P_{E}(\mathbf{w})$ for the beamformer (5) with the weight vector $\mathbf{w}$ is given in Appendix.

Stationary system. A linear antenna array with $n_{R}=4$ elements and a half-wavelength element spacing was employed to support $n_{T}=416-\mathrm{QAM}$ users. The angles of arrival for the four users were $10^{\circ}, 40^{\circ},-15^{\circ}$ and $-45^{\circ}$, respectively. The simulated stationary channels were $A_{m}=1+j 0,1 \leq m \leq 4$. The number of pilot symbols for the semi-blind scheme was

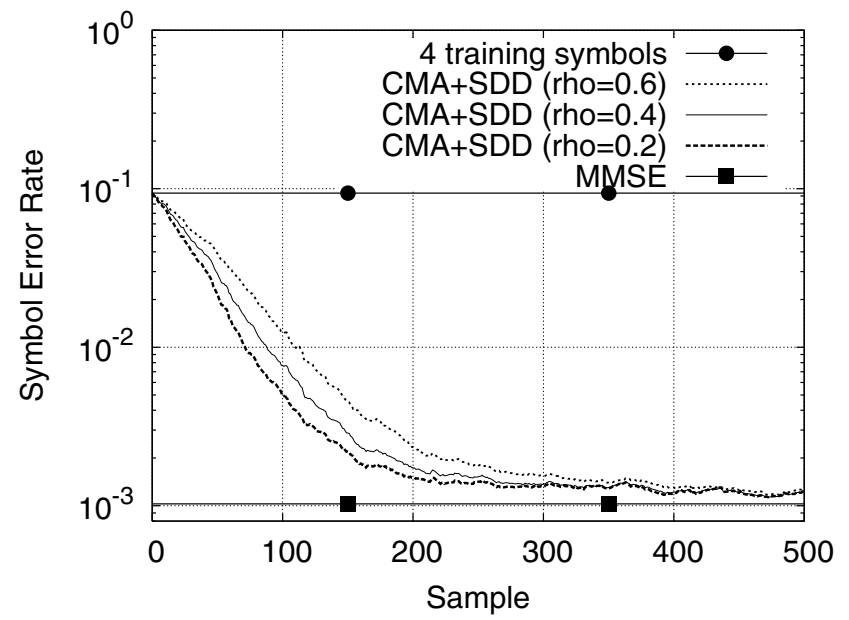

Fig. 3. Learning curves of the concurrent CMA and SDD scheme in terms of the SER average over ten different runs for the stationary system of fourelement array supporting four 16-QAM users, given the SNR of $18 \mathrm{~dB}$, $\mu_{\mathrm{CMA}}=4 \times 10^{-6}, \mu_{\mathrm{SDD}}=4 \times 10^{-4}$ and three values of cluster width $\rho$. 
$K=4$. Fig. 2 compares the desired-user SER performance of the proposed semi-blind beamforming scheme with those of the training-based beamforming given different numbers of training symbols, using the MMSE beamforming computed with the perfect channel knowledge as the benchmark. In the training-based adaptive beamforming, given $K$ training symbols, the LS estimate of the beamformer's weight vector was obtained according to (7), and the resulting desired-user SER was calculated. The performance of the training-based beamforming with $K=64$ training symbols, not shown in Fig. 2, was similar to that of the semi-blind combined CMA and SDD beamforming. It can be seen from Fig. 2 that the semi-blind scheme with four training symbols can closely match the performance of the true MMSE beamforming, while the pure training-based scheme requires at least 64 training symbols to achieve a similar performance.

The convergence performance of the proposed semi-blind scheme was investigated. Given the SNR of $18 \mathrm{~dB}, K=4$ training pilots were first used to provide the initial beamforming weight vector according to (7). The appropriate values for the step size of the CMA as well as the step size of the SDD were found empirically, and they were chosen to be $\mu_{\mathrm{CMA}}=4 \times 10^{-6}$ and $\mu_{\mathrm{SDD}}=4 \times 10^{-4}$, respectively. Fig. 3 plots the learning curves of the combined CMA and SDD adaptive algorithm in terms of the SER averaged over ten different runs, given three values of $\rho$. It is observed from Fig. 3 that, aiding by the four training pilots, the convergence rate of the concurrent CMA and SDD algorithm was much faster than the pure blind adaptive counterpart of [23], [24]. Furthermore, the proposed semi-blind scheme is capable of approaching the optimal MMSE solution, as can be seen in Fig. 3. Given the SNR of $18 \mathrm{~dB}, K=4$ training symbols were insufficient for the training-based beamformer, and the eye diagram of the beamformer's output constellation before the blind adaptation, i.e. with the weight vector $\mathbf{w}(0)$ of (7),

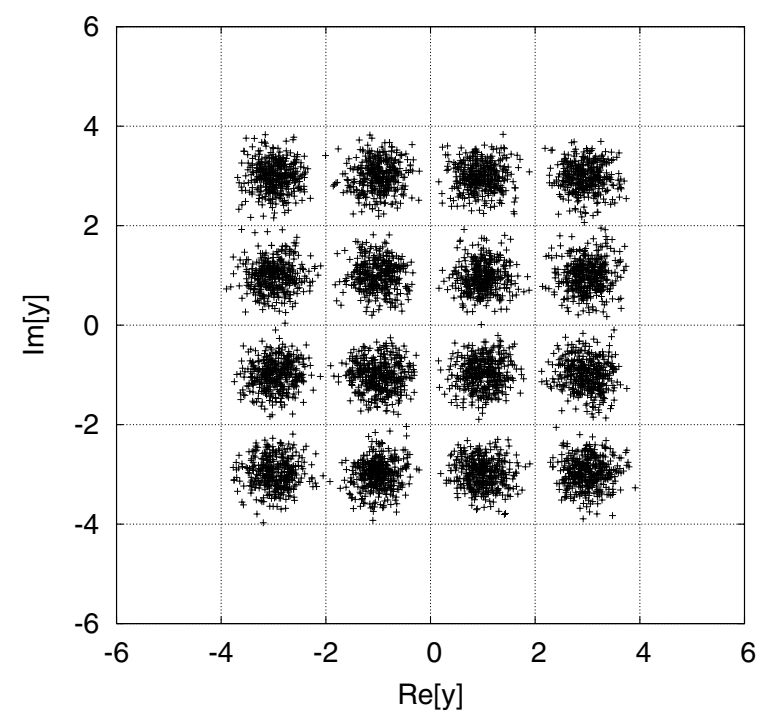

Fig. 4. The beamformer's output constellation after blind adaptation given SNR of $18 \mathrm{~dB}$ for the stationary system of four-element array supporting four 16-QAM users, shown with 6000 data points.

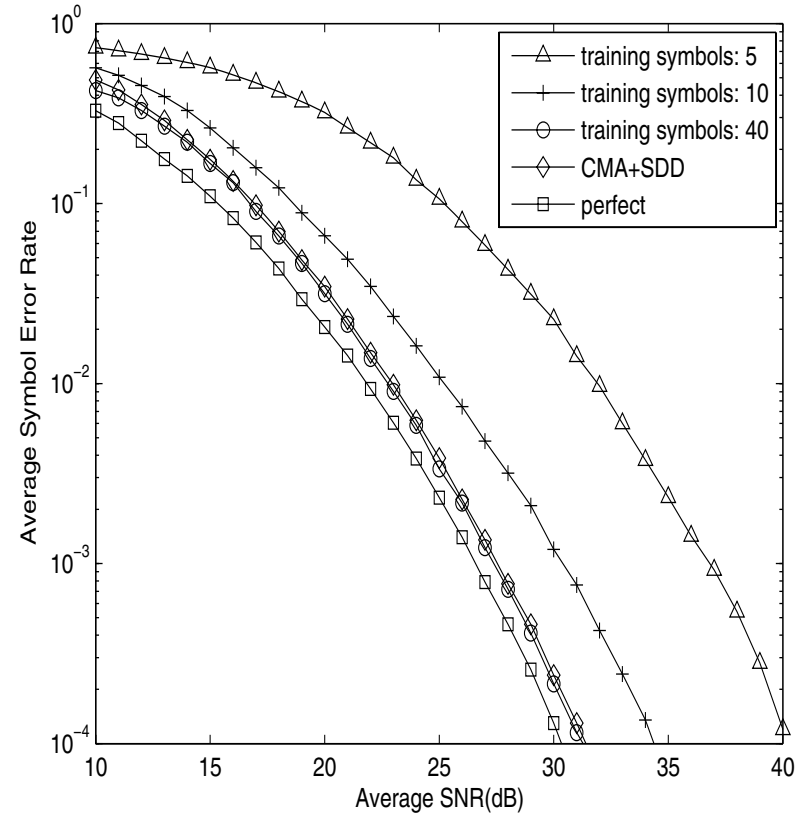

Fig. 5. Average symbol error rate performance of the proposed semi-blind beamforming scheme with five training symbols, in comparison with the cases of training only based on different numbers of training symbols and the MMSE beamforming with the perfect channel knowledge, averaged over 100 realisations of the flat-fading $5 \times 416$-QAM beamforming system.

was completely closed. By contrast, the beamformer's output constellation after blind adaptation is illustrated in Fig. 4, clearly showing that the eye was opened.

Flat fading system. A beamforming system with $n_{T}=4$, $n_{R}=5$ and the 16-QAM modulation scheme was simulated. The system's channel impulse response taps $h_{l, m}, 1 \leq l \leq 5$ and $1 \leq m \leq 4$, were uncorrelated complex-valued Gaussian processes with zero mean and $E\left[\left|h_{l, m}\right|^{2}\right]=1$, and the performance was averaged over 100 system realisations. The number of pilot symbols used for the semi-blind scheme was $K=5$. The average SER performance for the purely training based scheme with 5,15 and 40 training symbols, respectively, as well as the proposed semi-blind beamforming scheme with aid of 5 training symbols are shown in Fig. 5, in comparison with the achievable performance of the MMSE beamforming given the perfect channel knowledge. The step size of the CMA as well as the step size and cluster width of the SDD were empirically set to $\mu_{\mathrm{CMA}}=1 \times 10^{-7}$, $\mu_{\mathrm{SDD}}=2 \times 10^{-4}$ and $\rho=0.4$. The blind adaptive process was observed to achieve convergence typically within 200 to 400 samples. It can be seen from Fig. 5 that to achieve a similar performance as the semi-blind CMA-SDD scheme the training based scheme required 40 training symbols.

\section{COnCLusions}

A low-complexity semi-blind adaptive beamforming scheme has been proposed for wireless systems that employ high throughput QAM signalling. A minimum number of training symbols, equal to the number of receiver antenna-array's elements, is used to provide a rough LS estimate of the beamformer's weight vector for initialisation. The CMA aided 
SDD blind adaptive scheme is then adopted to adapt the beamformer. Our simulation study has confirmed that this semi-blind concurrent CMA and SDD algorithm converges much faster than its pure blind counterpart and the proposed semi-blind adaptive beamformer is capable of approaching the performance of the optimal MMSE beamforming solution.

\section{APPENDIX}

The analytical SER for the beamformer (5) with the weight vector $\mathbf{w}$ is given in [29]. Define the combined system response as $\mathbf{w}^{H} \mathbf{H}=\left[\begin{array}{llll}c_{1} & c_{2} & \cdots & c_{n_{T}}\end{array}\right]$, and assume that $c_{1}=c_{R_{1}}+$ $j c_{I_{1}}$ satisfies $c_{R_{1}}>0$ and $c_{I_{1}}=0$. The MMSE beamforming solution (6) meets this condition. For our proposed semi-blind beamformer, this condition is generally met, as it approaches the MMSE solution. If this condition is not satisfied, a rotation operation can always be performed on the weight vector to guarantee this condition [29]. The SER is expressed as

$$
P_{E}(\mathbf{w})=P_{E_{R}}(\mathbf{w})+P_{E_{I}}(\mathbf{w})-P_{E_{R}}(\mathbf{w}) P_{E_{I}}(\mathbf{w}),
$$

where $P_{E_{R}}(\mathbf{w})$ and $P_{E_{I}}(\mathbf{w})$ are the real-part and imaginarypart SERs, respectively. Note $\mathbf{x}(k)=\overline{\mathbf{x}}(k)+\mathbf{n}(k)$ and $y(k)=$ $\bar{y}(k)+e(k)$, where $e(k)$ is Gaussian distributed with zero mean and $E\left[|e(k)|^{2}\right]=2 \sigma_{n}^{2} \mathbf{w}^{H} \mathbf{w}$. The noise-free part $\bar{y}(k)$ takes values from the set $\mathcal{Y}$ that contains $N_{s}=M^{n_{T}}$ points. $\mathcal{Y}$ can be divided into the $M$ subsets conditioned on $s_{1}(k)$ as

$$
\mathcal{Y}^{(l, q)} \triangleq\left\{\bar{y}_{i}^{(l, q)} \in \mathcal{Y}, 1 \leq i \leq N_{s b}: s_{1}(k)=s_{l, q}\right\},
$$

for $1 \leq l, q \leq \sqrt{M}$, where the size of $\mathcal{Y}^{(l, q)}$ is $N_{s b}=$ $N_{s} / M$. The subset $\mathcal{Y}^{(l, q)}$ is completely specified by the system channel matrix $\mathbf{H}$. The SER $P_{E}(\mathbf{w})$ can be calculated based on a single subset $\mathcal{Y}^{(l, q)}$ [29]. Expressing $\bar{y}_{i}^{(l, q)}=\bar{y}_{R_{i}}^{(l, q)}+j \bar{y}_{I_{i}}^{(l, q)}$, it can be shown that [29]

$$
\begin{aligned}
& P_{E_{R}}(\mathbf{w})=\gamma \frac{1}{N_{s b}} \sum_{i=1}^{N_{s b}} Q\left(g_{R_{i}}^{(l, q)}(\mathbf{w})\right), \\
& P_{E_{I}}(\mathbf{w})=\gamma \frac{1}{N_{s b}} \sum_{i=1}^{N_{s b}} Q\left(g_{I_{i}}^{(l, q)}(\mathbf{w})\right),
\end{aligned}
$$

where $\gamma=\frac{2 \sqrt{M}-2}{\sqrt{M}}, Q(u)=\frac{1}{\sqrt{2 \pi}} \int_{u}^{\infty} e^{-\frac{z^{2}}{2}} d z$,

$$
\begin{aligned}
& g_{R_{i}}^{(l, q)}(\mathbf{w})=\frac{\bar{y}_{R_{i}}^{(l, q)}-c_{R_{1}}\left(u_{l}-1\right)}{\sigma_{n} \sqrt{\mathbf{w}^{H} \mathbf{w}}}, \\
& g_{I_{i}}^{(l, q)}(\mathbf{w})=\frac{\bar{y}_{I_{i}}^{(l, q)}-c_{R_{1}}\left(u_{q}-1\right)}{\sigma_{n} \sqrt{\mathbf{w}^{H} \mathbf{w}}} .
\end{aligned}
$$

\section{REFERENCES}

[1] J.H. Winters, J. Salz and R.D. Gitlin, "The impact of antenna diversity on the capacity of wireless communication systems," IEEE Trans. Communications, vol.42, no.2, pp.1740-1751, 1994.

[2] J. Litva and T.K.Y. Lo, Digital Beamforming in Wireless Communications. London: Artech House, 1996.

[3] L. C. Godara, "Applications of antenna arrays to mobile communications, Part I: Performance improvement, feasibility, and system considerations," Proc. IEEE, vol.85, no.7, pp.1031-1060, 1997.

[4] J.H. Winters, "Smart antennas for wireless systems," IEEE Personal Communications, vol.5, no.1, pp.23-27, 1998.
[5] J.S. Blogh and L. Hanzo, Third Generation Systems and Intelligent Wireless Networking - Smart Antenna and Adaptive Modulation. Chichester: John Wiley, 2002.

[6] A. Paulraj, R. Nabar and D. Gore, Introduction to Space-Time Wireless Communications. Cambridge: Cambridge University Press, 2003.

[7] A.J. Paulraj, D.A. Gore, R.U. Nabar and H. Bölcskei, "An overview of MIMO communications - A key to gigabit wireless," Proc. IEEE, vol.92, no.2, pp.198-218, 2004.

[8] L. Hanzo, S.X. Ng, T. Keller and W. Webb, Quadrature Amplitude Modulation: From Basics to Adaptive Trellis-Coded, Turbo-Equalised and Space-Time Coded OFDM, CDMA and MC-CDMA Systems, 2nd edition. Chichester, UK: John Wiley, 2004.

[9] IEEE 802.16 (2004). Air Interface for Fixed Broadband Wireless Access System, Section 8. PHY.

[10] B. Widrow, P.E. Mantey, L.J. Griffiths and B.B. Goode, "Adaptive antenna systems," Proc. IEEE, vol.55, pp.2143-2159, 1967.

[11] L.J. Griffiths, "A simple adaptive algorithm for real-time processing in antenna arrays," Proc. IEEE, vol.57, pp.1696-1704, 1969.

[12] S. Haykin, Adaptive Filter Theory. 3rd edition, Upper Saddle River, NJ: Prentice-Hall, 1996.

[13] J.J. Shynk and R.P. Gooch, "The constant modulus array for cochannel signal copy and direction finding," IEEE Trans. Signal Processing, vol.44, no.3, pp.652-660, 1996.

[14] J. Sheinvald, "On blind beamforming for multiple non-Gaussian signals and the constant-modulus algorithm," IEEE Trans. Signal Processing, vol.46, no.7, pp.1878-1885, 1998.

[15] A.-J. Van Der Veen, "Algebraic methods for deterministic blind beamforming," Proc. IEEE, vol.86, no.10, pp.1987-2008, 1998.

[16] K. Yang, T. Ohira, Y. Zhang and C.-Y. Chi, "Super-exponential blind adaptive beamforming," IEEE Trans. Signal Processing, vol.52, no.6, pp1549-1563, 2004.

[17] L. Tang, R.W. Liu, V.C. Soon and Y.F. Huang, "Indeterminacy and identifiability of blind identification," IEEE Trans. Circuits and Systems, vol.38, no.5, pp.499-509, 1991.

[18] Y. Inouye and R.W. Liu, "A system-theoretic foundation for blind equalization of an FIR MIMO channel system," IEEE Trans. Circuits and Systems Part I: Fundamental Theory and Applications, vol.49, no.4, pp.425-436, 2002.

[19] X. Mestre, M. Nájar, C. Antón and J.R. Fonollosa, "Adaptive beamforming for high bit rate services in the FDD mode of UTRA," in Proc. VTC1999-Fall, Sept.19-22, 1999, vol.4, pp.1951-1955.

[20] D. Bartolomé, A.I. Pézez-Neira and A. Pascual, "Blind and semiblind spatio-temporal diversity for OFDM systems," in Proc. ICASSP'02, May 13-17, 2002, vol.3, pp.2769-2772.

[21] R.A. Pacheco and D. Hatzinakos, "Blind (training-like) decoder assisted beamforming for DS-CDMA systems," in Proc. ICASSP'03, April 6-10, 2003, vol.4, pp.433-436.

[22] H. Zamiri-Jafarian, S. Bokharaiee-Najafee and S. Pasupathy, "EM based semi-blind beamforming algorithm in MIMO-OFDM systems," in Proc. 2007 IEEE Pacific Rim Conf. Communications, Computers and Signal Processing, Aug.22-24, 2007, pp.190-193.

[23] S. Chen and E.S. Chng, "Concurrent constant modulus algorithm and soft decision directed scheme for fractionally-spaced blind equalization," in Proc. ICC 2004 (Paris, France), June 20-24, 2004, vol.4, pp.23422346.

[24] S. Chen, A. Wolfgang and L. Hanzo, "Constant modulus algorithm aided soft decision directed scheme for blind space-time equalisation of SIMO channels," Signal Processing, vol.87, no.11, pp.2587-2599, 2007.

[25] D. Tse, and P. Viswanath, Fundamentals of Wireless Communication. Cambridge, U.K.: Cambridge University Press, 2005.

[26] D. Godard, "Self-recovering equalization and carrier tracking in twodimensional data communication systems," IEEE Trans. Communications, vol.COM-28, pp.1867-1875, 1980.

[27] J.R. Treichler and B.G. Agee, "A new approach to multipath correction of constant modulus signals," IEEE Trans. Acoustics, Speech and Signal Processing, vol.ASSP-31, no.2, pp.459-472, 1983.

[28] S. Chen, S. McLaughlin, P.M. Grant, and B. Mulgrew, "Multi-stage blind clustering equaliser," IEEE Trans. Communications, vol.43, no.3, pp.701-705, 1995.

[29] S. Chen, H.-Q. Du and L. Hanzo, "Adaptive minimum symbol error rate beamforming assisted receiver for quadrature amplitude modulation systems," in Proc. VTC2006-Spring (Melbourne, Australia), May 7-10, 2006, Vol.5, pp.2236-2240. 